\title{
Initial conditions for QCD evolution of double parton distributions
}

\section{Emilia LEWANDOWSKA*}

Institute of Nuclear Physics PAN

E-mail: emilia.lewandowskadifj.edu.pl

\section{Krzysztof GOLEC-BIERNAT}

Institute of Nuclear Physics PAN

E-mail: krzysztof.golec-biernateifj.edu.pl

Double parton distribution functions (DPDF) are used in the QCD description of double parton scattering. The DPDF evolve with hard scales through a relatively new QCD evolution equations and obey nontrivial sum rules: momentum and valence quark number sum rules. Based on a constructed numerical program, we present recent results on the QCD evolution of the DPDF. In particular, we discuss the problem how to specify initial conditions for the evolution equations which exactly fulfill the above mentioned sum rules. In current proposals, these rules are obeyed only approximately.

XXI International Workshop on Deep-Inelastic Scattering and Related Subjects 22-26 April, 2013

Marseilles, France

\footnotetext{
* Speaker.
} 


\section{Introduction}

Double parton distribution functions (DPDFs) are used in the description of double hard scattering. Their QCD evolution equations are known in the leading logarithmic approximation (LLA). The DPDFs obey nontrivial sum rules which are conserved by the evolution equations. The problem we address is how to specify initial conditions for the evolution equations which obey these

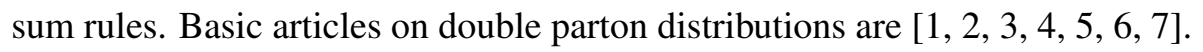

\section{Parton distribution functions}

In the single parton scattering (SPS), the final state of the hadron-hadron collision has been produced from only one hard interaction while in the double parton scattering, two hard subprocesses occur. For the description of the SPS we use the single parton distribution functions, $D_{f}(x, Q)$, while for the double parton scattering - the double parton distribution functions denoted by $D_{f_{1} f_{2}}\left(x_{1}, x_{2}, Q_{1}, Q_{2}\right)$. The DPDF depend on two parton flavours $f_{1}, f_{2}$ (including gluon), two parton momentum fractions $x_{1}, x_{2}$ and two hard scales $Q_{1}, Q_{2}$. The two parton momentum fractions obey the condition saying that the sum of partons' momenta cannot exceed the total nucleon momentum,

$$
x_{1}+x_{2} \leq 1
$$

\section{Evolution equations}

The general form of QCD evolution equations for single PDF is given by

$$
\partial_{t} D_{f}(x, t)=\sum_{f^{\prime}} \int_{0}^{1} d u \mathscr{K}_{f f^{\prime}}(x, u, t) D_{f^{\prime}}(u, t),
$$

with the evolution parameter $t=\ln \left(Q^{2} / Q_{0}^{2}\right)$. The integral kernels $\mathscr{K}_{f f^{\prime}}$ presented in above equation describe real and virtual parton emission. The real emission kernels take the following form

$$
\mathscr{K}_{f f^{\prime}}^{R}(x, u, t)=\frac{1}{u} P_{f f^{\prime}}\left(\frac{x}{u}, t\right) \theta(u-x)
$$

in which $P_{f f^{\prime}}$ are splitting functions computed perturbatively in QCD in powers of the strong coulpling constant $\alpha_{s}$ :

$$
P_{f f^{\prime}}(z, t)=\frac{\alpha_{s}(t)}{2 \pi} P_{f f^{\prime}}^{(0)}(z)+\frac{\alpha_{s}^{2}(t)}{(2 \pi)^{2}} P_{f f^{\prime}}^{(1)}(z)+\ldots
$$

After including the splitting functions, we find the well known DGLAP evolution equations for the single PDFs:

$$
\partial_{t} D_{f}(x, t)=\sum_{f^{\prime}} \int_{x}^{1} \frac{d z}{z} P_{f f^{\prime}}(z, t) D_{f^{\prime}}\left(\frac{x}{z}, t\right)-D_{f}(x, t) \sum_{f^{\prime}} \int_{0}^{1} d z z P_{f^{\prime} f}(z, t) .
$$


In the leading logarithmic approximation, the evolution equations of the DPDFs (for equal two hard scales, $\left.Q_{1}=Q_{2}, \equiv Q\right)$ have the following form

$$
\begin{aligned}
\partial_{t} D_{f_{1} f_{2}}\left(x_{1}, x_{2}, t\right) & =\sum_{f^{\prime}} \int_{0}^{1-x_{2}} d u \mathscr{K}_{f_{1} f^{\prime}}\left(x_{1}, u, t\right) D_{f^{\prime} f_{2}}\left(u, x_{2}, t\right) \\
& +\sum_{f^{\prime}} \int_{0}^{1-x_{1}} d u \mathscr{K}_{f_{2} f^{\prime}}\left(x_{2}, u, t\right) D_{f_{1} f^{\prime}}\left(x_{1}, u, t\right) \\
& +\sum_{f^{\prime}} \mathscr{K}_{f^{\prime} \rightarrow f_{1} f_{2}}^{R}\left(x_{1}, x_{1}+x_{2}, t\right) D_{f^{\prime}}\left(x_{1}+x_{2}, t\right) .
\end{aligned}
$$

The upper integration limits reflect the condition (2.1). The third term constains the single PDFs and because of that eqs. (B.5) and (B.D) have to be solved together. That is why the initial conditions for both the double and single PDFs have to be specified at some initial scale $Q_{0}$.

\section{Sum rules}

The DGLAP evolution equations preserve the momentum sum rule for the single PDFs:

$$
\sum_{f} \int_{0}^{1} d x x D_{f}(x, Q)=1 .
$$

By analogy, one finds the momentum sum rule for the DPDF

$$
\sum_{f_{1}} \int_{0}^{1-x_{2}} d x_{1} x_{1} \frac{D_{f_{1} f_{2}}\left(x_{1}, x_{2}, Q\right)}{D_{f_{2}}\left(x_{2}, Q\right)}=\left(1-x_{2}\right) .
$$

The ratio of the double and single PDFs in the above is conditional probability to find a parton with the momentum fraction $x_{1}$, while the second parton characteristics are fixed. It is clearly seen that the new momentum sum rule relates the double and single PDFs

$$
\sum_{f_{1}} \int_{0}^{1-x_{2}} d x_{1} x_{1} D_{f_{1} f_{2}}\left(x_{1}, x_{2}, Q\right)=\left(1-x_{2}\right) D_{f_{2}}\left(x_{2}, Q\right) .
$$

The valence number sum rule for the single quark distributions has the well known form

$$
\int_{0}^{1} d x\left\{D_{q_{i}}(x, Q)-D_{\bar{q}_{i}}(x, Q)\right\}=N_{i}
$$

For the DPDFs, one finds a similar relation with three cases for each value of the second parton flavour [3]

$$
\int_{0}^{1-x_{2}} d x_{1}\left\{D_{q_{i} f_{2}}\left(x_{1}, x_{2}, Q\right)-D_{\bar{q}_{i} f_{2}}\left(x_{1}, x_{2}, Q\right)\right\}= \begin{cases}N_{i} D_{f_{2}}\left(x_{2}, Q\right) & \text { for } f_{2} \neq q_{i}, \bar{q}_{i} \\ \left(N_{i}-1\right) D_{f_{2}}\left(x_{2}, Q\right) & \text { for } f_{2}=q_{i} \\ \left(N_{i}+1\right) D_{f_{2}}\left(x_{2}, Q\right) & \text { for } f_{2}=\bar{q}_{i}\end{cases}
$$

It is important to emphasize once more that the momentum and the valence number sum rules are conserved by the evolution equations once they are imposed at the initial scale. 


\section{Initial conditions}

In order to solve the evolution equations we need to specify initial conditions. For practical reason, their form is to built out of the existing single PDFs. For example, the symmetric input [1], []] is built out of the two single PDFs and some correlation factor:

$$
D_{f_{1} f_{2}}\left(x_{1}, x_{2}\right)=D_{f_{1}}\left(x_{1}\right) D_{f_{2}}\left(x_{2}\right) \frac{\left(1-x_{1}-x_{2}\right)^{2}}{\left(1-x_{1}\right)^{2+n_{1}}\left(1-x_{2}\right)^{2+n_{2}}}
$$

This input is symmetric under the parton interchange, $D_{f_{1} f_{2}}\left(x_{1}, x_{2}\right)=D_{f_{2} f_{1}}\left(x_{2}, x_{1}\right)$, and also positive definite (if single PDFs are). However, this input violates the sum valence number rules. The graphical ilustration of the sum rule violation by the symmetric input is shown below by plotting the ratio of the r.h.s. to l.h.s. for the sum rules (4.3) and (4.5)).
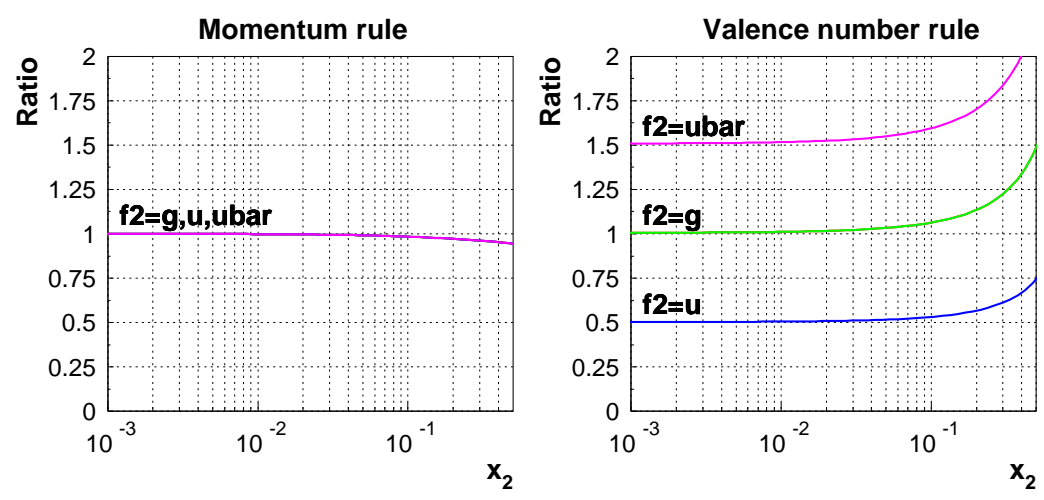

How to exacly fulfill those sum rules? In order to obey the momentum sum rule we could use the asymmetric ansatz:

$$
D_{f_{1} f_{2}}\left(x_{1}, x_{2}\right)=\frac{1}{1-x_{2}} D_{f_{1}}\left(\frac{x_{1}}{1-x_{2}}\right) \cdot D_{f_{2}}\left(x_{2}\right) .
$$

To fulfill the valence number sum rule we need to introduce corrections for identical quark flavours and antiflavours

$$
\begin{aligned}
& D_{f_{i} f_{i}}\left(x_{1}, x_{2}\right)=\frac{1}{1-x_{2}}\left\{D_{f_{1}}\left(\frac{x_{1}}{1-x_{2}}\right)-\frac{1}{2}\right\} D_{f_{1}}\left(x_{2}\right) \\
& D_{f_{i} \bar{f}_{i}}\left(x_{1}, x_{2}\right)=\frac{1}{1-x_{2}}\left\{D_{f_{1}}\left(\frac{x_{1}}{1-x_{2}}\right)+\frac{1}{2}\right\} D_{\bar{f}_{1}}\left(x_{2}\right)
\end{aligned}
$$

It is important that those corrections do not spoil the momentum sum rule. However, the DPDFs for identical flavours are not generally positive definite because of the factor $-1 / 2$. This is the price to pay for the construction with single PDFs. 
The graphical comparison of the symmetric and asymmetric inputs is shown below.

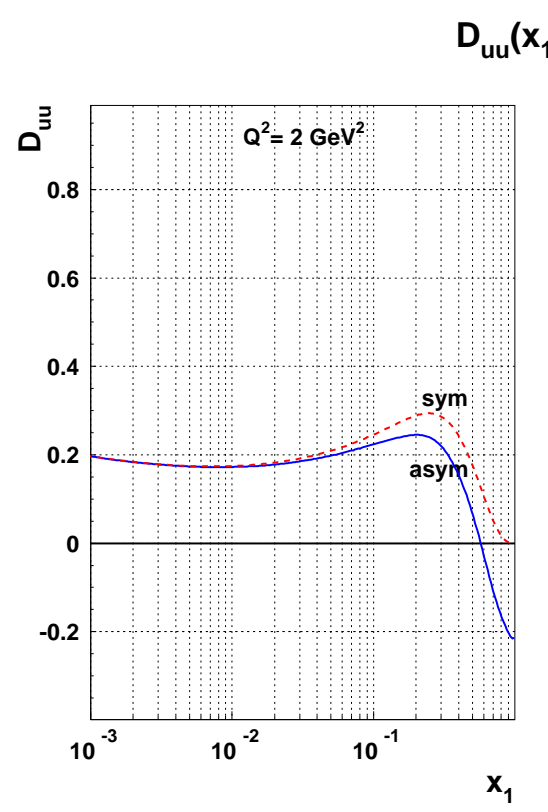

$D_{u u}\left(x_{1}, x_{2}=10^{-3}\right)$

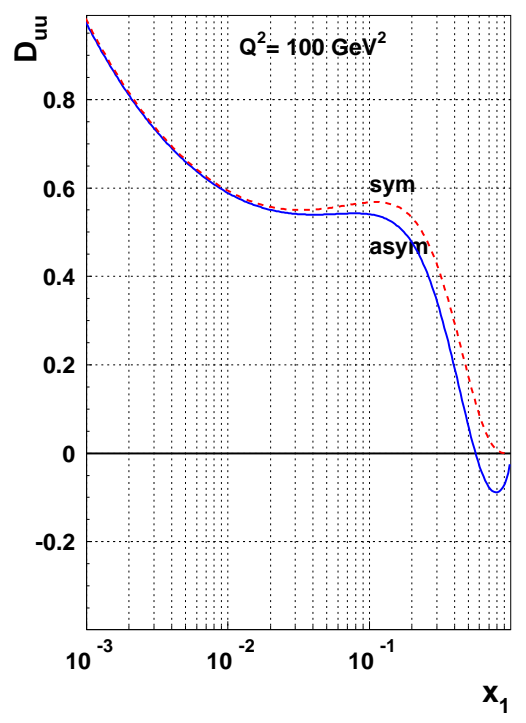

For the distributions $D_{u u}$ both inputs give similar results in the small $x_{1}$ region, while for large $x_{1}$ there are differences between them because of the lack of positive definitness of the asymmetric input. The same results are found for the evolved distribution.

For $D_{u \bar{u}}$, both distributions are positive but there are differences between them at the large $x_{1}$ :

$$
D_{\text {uubar }}\left(x_{1}, x_{2}=10^{-3}\right)
$$
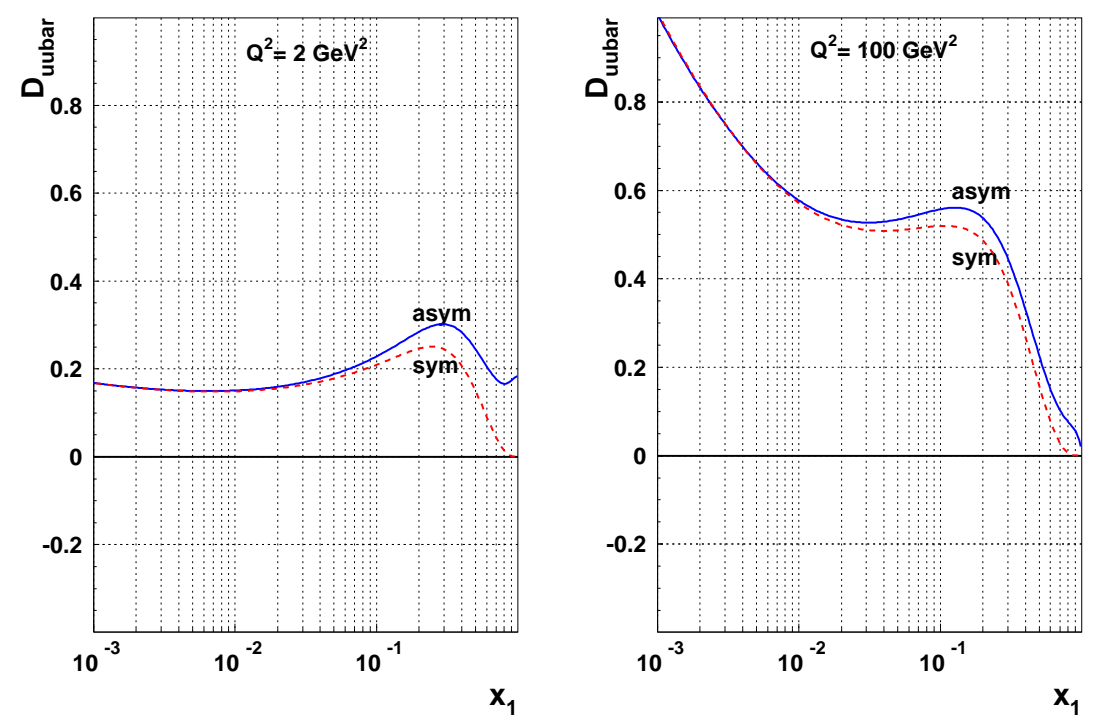
And finally, for the $D_{g u}$ distributions both inputs give the same results in the whole $x_{1}$ domain.

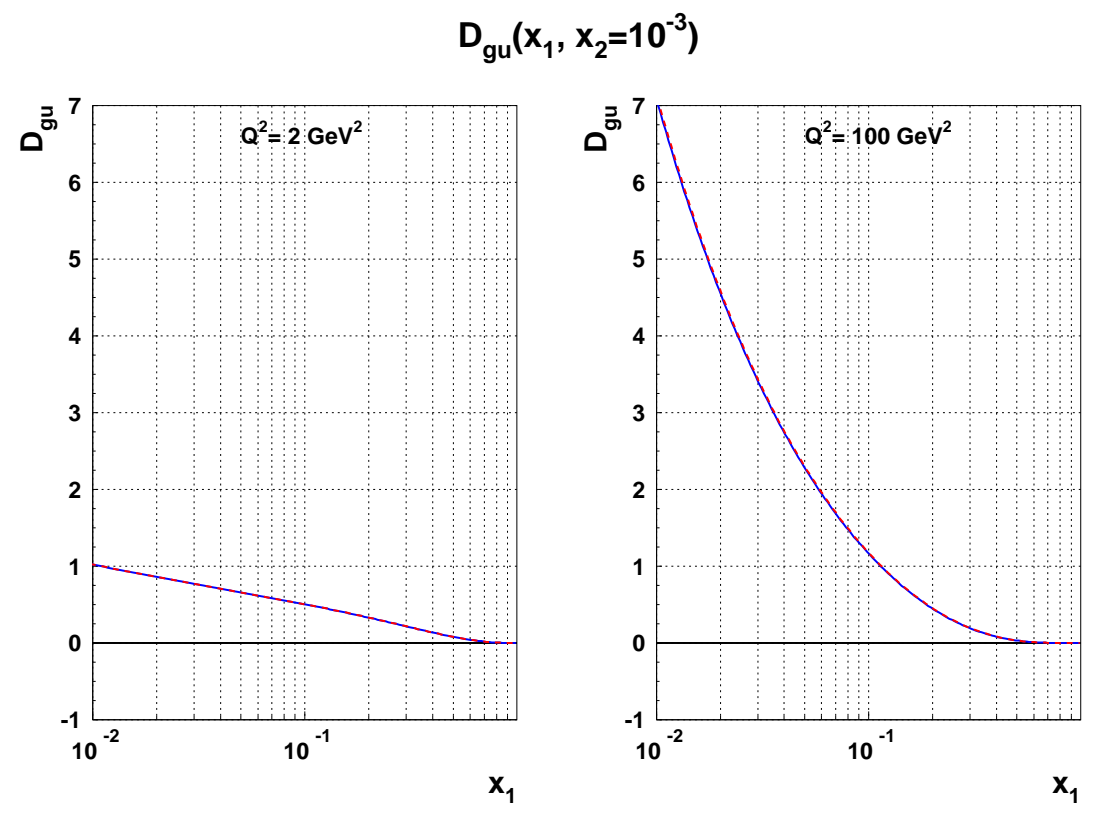

\section{Summary}

The specification of the initial conditions for evolution of the DPDFs is not simple. The symmetric input gives parton symmetry and positivity, but does not exactly fulfill sum rules. In the other hand, the asymmetric input obeys them exactly, but is not positive definite. There is also an alternative solution: we could specify positive initial double distributions and then generate the single PDF using sum rules. However, at present time, there is no experimental knowledge of the DPDF. For small parton momentum fractions, the factorized form $D_{f_{1} f_{2}}\left(x_{1}, x_{2}, Q\right)=D_{f_{1}}\left(x_{1}, Q\right) D_{f_{2}}\left(x_{2}, Q\right)$ is a good approximation, however, the problem occurs for large $x$ values $\left(>10^{-2}\right)$.

\section{Acknowledgement}

This work was supported by the Polish NCN grants DEC-2011/01/B/ST2/03915 and DEC2012/05/N/ST2/02678.

\section{References}

[1] A. M. Snigirev, Phys. Rev. D68, 114012 (2003), [hep-ph/0304172].

[2] V. L. Korotkikh and A. M. Snigirev, Phys. Lett. B594, 171 (2004), [hep-ph/0404155].

[3] J. R. Gaunt and W. J. Stirling, JHEP 03, 005 (2010), [0910.4347].

[4] F. A. Ceccopieri, Phys. Lett. B697, 482 (2011), [1011.6586].

[5] J. R. Gaunt and W. J. Stirling, JHEP 1106, 048 (2011), [1103.1888].

[6] M. Ryskin and A. Snigirev, Phys.Rev. D83, 114047 (2011), [1103.3495].

[7] M. Diehl and A. Schafer, Phys. Lett. B698, 389 (2011), [1102.3081]. 